\title{
LOS GLACIARES ROCOSOS DE SIERRA NEVADA Y SU SIGNIFICADO PALEOCLIMÁTICO. UNA PRIMERA APROXIMACIÓN
}

\author{
B. PALADE ${ }^{(1)}$ * D. PALACIOS ESTREMERA ${ }^{(2)}$, A. GÓMEZ ORTIZ ${ }^{(3)}$ \\ ${ }^{1}$ Centro de Satélites de la Unión Europea (EUSC), Torrejón de Ardoz \\ ${ }^{2}$ Departamento de Análisis Geográfico Regional y Geografía Física, \\ Universidad Complutense de Madrid \\ ${ }^{3}$ Departamento de Geografía Física y Análisis Geográfico Regional. \\ Universidad de Barcelona
}

RESUMEN. En este trabajo se han identificado y cartografiado 36 glaciares rocosos en el sector occidental de Sierra Nevada. Todos los glaciares rocosos se incluyen en la categoría de los glaciares rocosos de talud. Exceptuando el caso del glaciar rocoso del Corral del Veleta que aún preserva ciertas masas de hielo interior, el resto de los glaciares rocosos han sido clasificados como formas relictas. La información cartográfica ha sido tratada en un Sistema de Información Geográfica (GIS) y los resultados indican que los glaciares rocosos se localizan entre los 2460 m y 3360 m de altitud, tienen una pendiente media de $21^{\circ}$ y prefieren las orientaciones con menor radiación, aunque también abundan en la cara sur de la Sierra, cuando las condiciones de acumulación de nieve así lo propician. La presente localización altitudinal de los glaciares rocosos indica que se formaron con una temperatura de $6^{\circ} \mathrm{C}$ inferior a la actual. Dos de los glaciares rocosos han sido datados de forma absoluta a través del isótopo cosmogénico ${ }^{36} \mathrm{Cl}$, así como un umbral con pulimento glaciar donde se asienta uno de ellos. Los resultados muestran que los glaciares rocosos se formaron justo después de la retirada de los hielos glaciares (hace 14 a 15 ka) y pudieron algunos sobrevivir hasta ya avanzado el Holoceno (entre hace 8 y $7 \mathrm{ka}$ ). El actual glaciar rocoso del Corral del Veleta es un nuevo ejemplo de formación de glaciares rocosos, después de la deglaciación de paredes muy inestables, en este caso al final de la Pequeña Edad de Hielo.

Palaeoclimatic significance of rock glaciers in Sierra Nevada. A first approach

ABSTRACT. This paper provides an inventory of 36 rock glaciers located in the western part of Sierra Nevada mountains. All the forms fall in the category of talus rock glaciers. All the rock glaciers were classified as relict except the rock 
glacier situated in Corral del Veleta which still preserves certain amount of ice in its interior. The cartographic information was included and analyzed within a Geographical Information System (GIS) and the results show that the rock glaciers in Sierra Nevada are located between 2460 and $3360 \mathrm{~m}$ a.s.l., on slopes of $21^{\circ}$ on average that receive low amounts of solar radiation, although due to particular wind direction and snow accumulation conditions in Sierra Nevada, there is a significant number of rock glaciers lying on the southern slopes. The present altitudinal distribution of rock glaciers suggests average temperatures $6^{\circ} \mathrm{C}$ lower than today at the time of their initiation. Two rock glaciers as well as one abrasion surface were sampled and dated using the ${ }^{36} \mathrm{Cl}$ cosmogenic exposure method. The results show that the rock glaciers developed immediately after the Pleistocene glaciers retreated (14 - $15 \mathrm{ka}$ ) and were still active throughout the first part of the Holocene (8-7 $\mathrm{ka}$ ). The rock glacier situated in the Corral del Veleta is a recent example of rock glacier formation in a deglaciated environment during the final phase of the Little Ice Age.

Palabras clave: glaciares rocosos, permafrost, paleoclimas, dataciones cosmogénicas, Sierra Nevada.

Key words: rock glaciers, permafrost, paleoclimates, cosmogenic dating, Sierra Nevada.

Enviado el 26 de agosto.de 2010 Aceptado el 10 de noviembre de 2010

*Correspondencia: Centro de Satélites de la Unión Europea (EUSC), Apartado de Correos 511. 28850-Torrejón de Ardoz (Madrid). E-mail: b.palade@eusc.europa.eu

\section{Introducción}

Los glaciares rocosos forman parte del paisaje de cumbres de Sierra Nevada, en concreto de su sector occidental que fue donde quedó recluido el glaciarismo cuaternario. De manera precisa no se identifican como tales hasta 1965, que es cuando Messerli los distingue como formas de relieve emblemáticas del relieve periglaciar, bajo la denominación de "Wallsysteme" (Messerli, 1965) y después Lhenaff (1977) atribuyéndolas a periodos finiglaciares. Antes de ellos, como tales, se dice poco, a lo sumo alusiones a formaciones morrénicas encerradas en el interior de los circos (Paschinger, 1957). Los glaciares rocosos nevadenses han venido permaneciendo un tanto desconocidos en lo que se refiere a sus orígenes y mecanismos de formación, así como a su significado paleoambiental en la historia geomorfológica reciente de Sierra Nevada. Fue en la segunda mitad de la década de los ochenta del siglo pasado cuando comienzan a despertar interés, en gran manera debido a la atención que ya se les había prestado a los glaciares rocosos del Pirineo Oriental a partir de la segunda mitad de 1970 (Soutadé, 1980; Serrat, 1979; Gómez Ortiz, 1987a; Serrano et al., 1999). 
El primer estudio monográfico sobre glaciares rocosos de Sierra Nevada se debió a Soria y Soria (1986), que tras describirlos y proponer una clasificación morfológica los instalan cronológicamente en las fases frías tardiglaciares. Posteriormente, desde 1987, estas formas de relieve han venido siendo motivo de atención continuada en trabajos sobre morfología glaciar y periglaciar de la Sierra (Gómez Ortiz, 1987b; Gómez Ortiz et al., 2002) aportándose de ellas información relativa a sus mecanismos de formación, morfologías y modelados e instalación en el tiempo a partir de los datos polínicos de la turbera de Padul (Flörschutz et al. 1971; Pons et al. 1988). Interés particular tiene también la elaboración de su cartografía geomorfológica (Gómez Ortiz et al., 2002).

De acuerdo con la mayoría de los últimos trabajos sobre permafrost (Degenhardt, 2009) los glaciares rocosos relictos representan una relevante fuente de información paleoclimática para el estudio de la evolución reciente del relieve alpino, posterior al último máximo avance glaciar. En tal sentido, hay que señalar que la presencia de estos glaciares rocosos en las cadenas montañosas mediterráneas viene a marcar la antigua extensión de la zona criogénica dominada por los procesos periglaciares generalizados.

Un aspecto clave de los trabajos que tratan el tema del permafrost de montaña es la relación entre la formación y actividad de los glaciares rocosos y las fases de glaciación/ deglaciación pleistocenas. En este contexto, algunos trabajos han intentado utilizar los glaciares rocosos como fuente de información paleoclimática (Kerschner, 1983). De esta manera, diversos estudios proponen aumentos de temperatura drásticos en las montañas mediterráneas, desde que grandes masas de glaciares rocosos estaban activos hasta la actualidad: $6,4^{\circ} \mathrm{C}$ para los Alpes de Albania (Palmentola et al., 1995), 8-9 ${ }^{\circ} \mathrm{C}$ para las montañas Pindus (Hughes et al., 2006) y hasta 9,5-10,5 ${ }^{\circ} \mathrm{C}$ para el Atlas (Hughes et al., 2004). Trabajos llevados a cabo en los Alpes de Suiza (Frauenfelder y Kääb, 2000) indican aumentos más moderados de temperatura de sólo $3-4^{\circ} \mathrm{C}$ desde la formación de los glaciares rocosos hasta el presente. Creemos que estas diferencias pueden tener su origen en la diferente interpretación sobre la edad real de los glaciares rocosos, a causa de la falta de dataciones absolutas.

El conocimiento de la extensión de la glaciación pleistocena en Sierra Nevada esta bien fundamentada en la actualidad (Gómez Ortiz et al., 2002); sin embargo, existen muy pocos datos con respecto a la distribución del permafrost al final del Pleistoceno. A través de la cartografía y el establecimiento de los límites altitudinales de los glaciares rocosos fósiles es posible reconstruir los antiguos límites del permafrost en el momento de su formación (Frauenfelder y Kääb, 2000). El objetivo de este trabajo es aplicar este método en Sierra Nevada y obtener dataciones absolutas por análisis de isótopos cosmogénicos del momento en que estos glaciares rocosos quedaron inmovilizados e inactivos. De esta manera se puede comparar la situación climática actual con aquella otra paleoclimática en la que se formaron los glaciares rocosos, cuando el permafrost se extendía por todo el área de cumbres de Sierra Nevada occidental. Este contraste puede resultar especialmente interesante si tenemos en cuenta que actualmente se ha detectado permafrost discontinuo en localidades aisladas de Sierra Nevada (interior de los circos y posiblemente en altiplanicies cimeras) (TERRADAT-LTD \& ETD, 1998; Tanarro et 
al., 2001). Este hecho, junto a las evidencias de flujo en glaciares rocosos y coladas de bloques asentadas en masas heladas en el seno del Corral del Veleta (Gómez Ortiz et al., 2003), vienen a fijar en las cumbres de Sierra Nevada el límite meridional actual del permafrost discontinuo en las montañas europeas. El conocer cuándo y en qué circunstancias climáticas se formaron estos glaciares rocosos, fósiles en la actualidad, puede aportar una información de máximo interés al conocimiento de la evolución climática europea a finales del Pleistoceno y principios del Holoceno.

\section{2. Área de estudio}

El área investigada ocupa un total de $40 \mathrm{~km}^{2}$ y se circunscribe en la parte centrooccidental de las cumbres de Sierra Nevada (Fig. 1). Ésta coincide con el sector más elevado del macizo (Mulhacén, 3482 m; Veleta, 3398 m), incluyendo las cabeceras de los ríos Veleta, Río Seco y Dílar, encarados al sur y noroeste y Guarnón, Valdeinfierno y Valdecasillas orientados al norte, todos ellos antiguos circos glaciares. Estas cabeceras de ríos (barrancos) albergan en su seno diferentes generaciones de glaciares rocosos.

Según Salvador Franch et al. (2010), en la zona de cumbres de Sierra Nevada las temperaturas medias anuales, a partir de 3300 metros, son negativas $\left(-0,4^{\circ} \mathrm{C}\right.$, a $\left.3398 \mathrm{~m}\right)$. $\mathrm{El}$ gradiente térmico vertical es de $0,48^{\circ} \mathrm{C}$ en la vertiente meridional y $0,61^{\circ} \mathrm{C}$ en la vertiente septentrional (Rodríguez Martínez et al., 1981). La fluctuación térmica diaria del

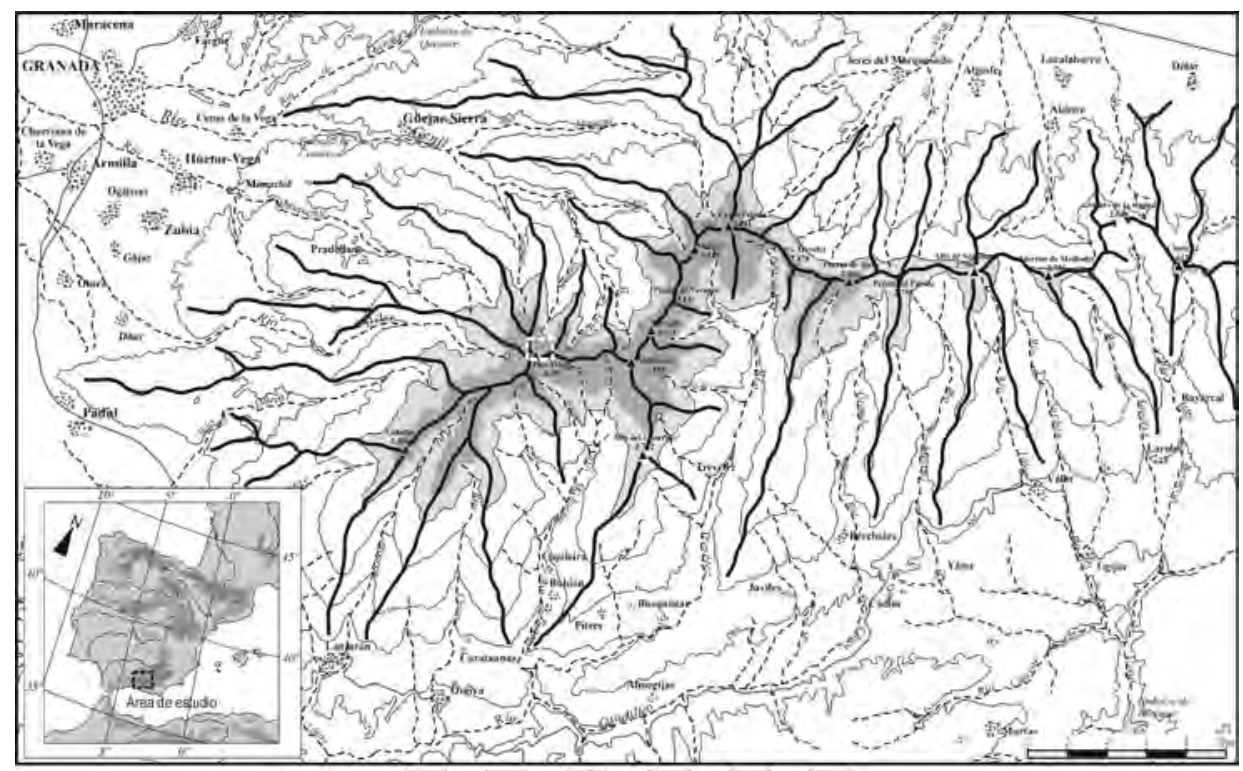

局, E, F, F, D, 口,

Figura 1. Localización de Sierra Nevada. 
aire entre los valores extremos es muy importante. En tal sentido, es destacable señalar que el valor máximo anual es del orden de $56,4^{\circ} \mathrm{C}$ y de $48,2^{\circ} \mathrm{C}$ en $\operatorname{los}-5 \mathrm{~cm}$ del suelo (Salvador Franch et al., 2010). Las precipitaciones superan los $775 \mathrm{~mm}$ a una altura de $2550 \mathrm{~m}$, de las que el 40\% son en forma de nieve (Montávez et al., 1996), pero su reparto mensual es desigual, particularmente en verano que recibe menos del $10 \%$ del total anual. Sierra Nevada se encuentra mayoritariamente bajo la influencia de la circulación atmosférica de origen atlántico lo que determina una superior humedad y precipitación en las vertientes de exposición norte y oeste. Pero, al mismo tiempo, y como respuesta a la dominancia de los vientos del cuarto cuadrante, la acumulación de nieve resulta muy abundante en las en laderas expuestas al este, sureste y sur, en su tránsito con las planicies más cimeras.

La zona de cumbres de la Sierra está labrada en el denominado complejo NevadoFilábride y la zona de estudio se incluye en el manto del Veleta (Díaz de Federico et al., 1980). El sustrato litológico de este manto está constituido, mayoritariamente, por sucesiones de diferentes bancos de rocas metamórficas muy tectonizadas y muy friables. Los materiales dominantes son los micaesquistos grafitosos, feldespáticos y anfibolíticos, las cuarcitas y los ortogneises (Puga et al., 2007). Se trata de rocas muy frágiles a su fragmentación mecánica y alteración química, lo que explica los gruesos amontonamientos de bloques que se almacenan al pie de cornisas y escarpes así como los espesores de sedimentos que definen el tapizado de laderas y vertientes a cotas más bajas.

El área de estudio se encuentra en su totalidad modelada por la glaciación cuaternaria (Gómez et al., 2001). De acuerdo con la mayoría de las investigaciones, los glaciares cuaternarios dejaron sus huellas en el relieve de Sierra Nevada en, al menos, dos periodos (Paschinger, 1957; Messerli, 1965; Lhenaff, 1977) habiendo podido alcanzar cotas de 1400 m en el valle de Lanjarón (Gómez et al., 2002). Sin embargo, las formas glaciares de Sierra Nevada quedan recluidas en las alturas y encerradas en el seno de los barrancos, pues la localización latitudinal de la Sierra y las condiciones climáticas regionales debieron limitar el desarrollo de las masas glaciares. Además, la vulnerabilidad de los micaesquistos a la meteorización, unido a los altos valores de energía del relieve, propiciaron la rápida erosión de los depósitos morrénicos dificultando su reconstrucción en el espacio glaciado. Simultáneamente a la retirada de las lenguas glaciares y posterior aislamiento del hielo en las cumbres, de manera más precisa en los circos de mayor altura, el ambiente de Sierra Nevada asistió a la rápida expansión generalizada de los procesos periglaciares y al desarrollo de sus formas más espectaculares: los glaciares rocosos.

La Pequeña Edad de Hielo también tuvo su incidencia en la Sierra, en particular en los circos de la vertiente norte situados a mayor altitud. De entre ellos, destaca el Corral del Veleta (en la cabecera del valle de Guarnón) que albergó "el glaciar europeo más meridional" (Boissier, 1845) y que perduró, ya muy reducido, hasta mediados de siglo XX (Gómez Ortiz et al., 2008) (Fig. 2). Su presencia queda bien documentada a través de escritos de época, a partir de mediados del siglo XVIII (Gómez Ortiz et al., 2006). En la actualidad, el Corral del Veleta se distingue por su singularidad morfológica, al guardar 


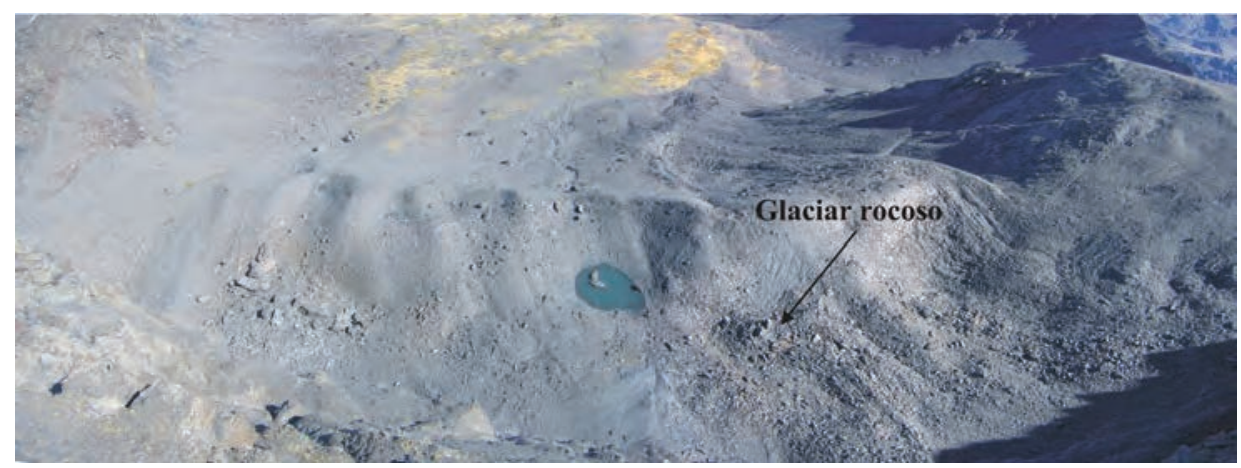

Figura 2. Formas de modelado en la base del cuenco del Corral del Veleta.

registros importantes de las últimas fases frías, a partir del Tardiglaciar convirtiéndose, así, en un lugar privilegiado para el estudio de los procesos fríos actuales en áreas de montaña. Además, resulta también singular por la morfodinámica fría actual que lo modela, con procesos de degradación de hielo glaciar fósil y permafrost y, asociado a ello, la construcción de importantes coladas, lóbulos de piedras e incipientes glaciares rocosos (Gómez Ortiz et al., 2008).

\section{Metodología}

El estudio preliminar de los glaciares rocosos de Sierra Nevada se ha desarrollado en dos etapas. En una primera, se llevó a cabo un inventario de estas formaciones del sector centro-occidental. Su identificación, localización y cartografía se ha realizado mediante fotointerpretación (vuelo 2001-2002, a una resolución espacial de 0,5 m) y posterior reconocimiento de campo. En una segunda etapa, se relacionó la distribución espacial de los glaciares rocosos identificados con diferentes variables topoclimáticas como la altitud, orientación y radiación solar. Para ello, previamente, se levantó el modelo digital de elevaciones (MDE) a partir de la topografía a escala 1:10.000 de la Junta de Andalucía (2005), obteniéndose una resolución de 10 metros (Fig. 3). El mapa de la radiación solar incidente sobre la superficie de los glaciares rocosos ha sido generado a partir del MDE para la estación libre de nieve entre mayo - noviembre. Sobre la cartografía producida se llevó a cabo un análisis morfométrico y morfológico con el fin de poder interpretar mejor las características que definen el ambiente propio de los glaciares rocosos cartografiados. Como último paso, se relacionó la actual distribución espacial de estas morfologías con la antigua extensión probable del permafrost discontinuo en el macizo y se realizó un cálculo previo de las diferencias térmicas entre la época de actividad de los glaciares rocosos y el presente. El tratamiento geomorfológico y morfométrico de los glaciares rocosos, incluyendo identificación y análisis espacial de las formas encontradas se realizó dentro del entorno ArcGIS 9.3. 


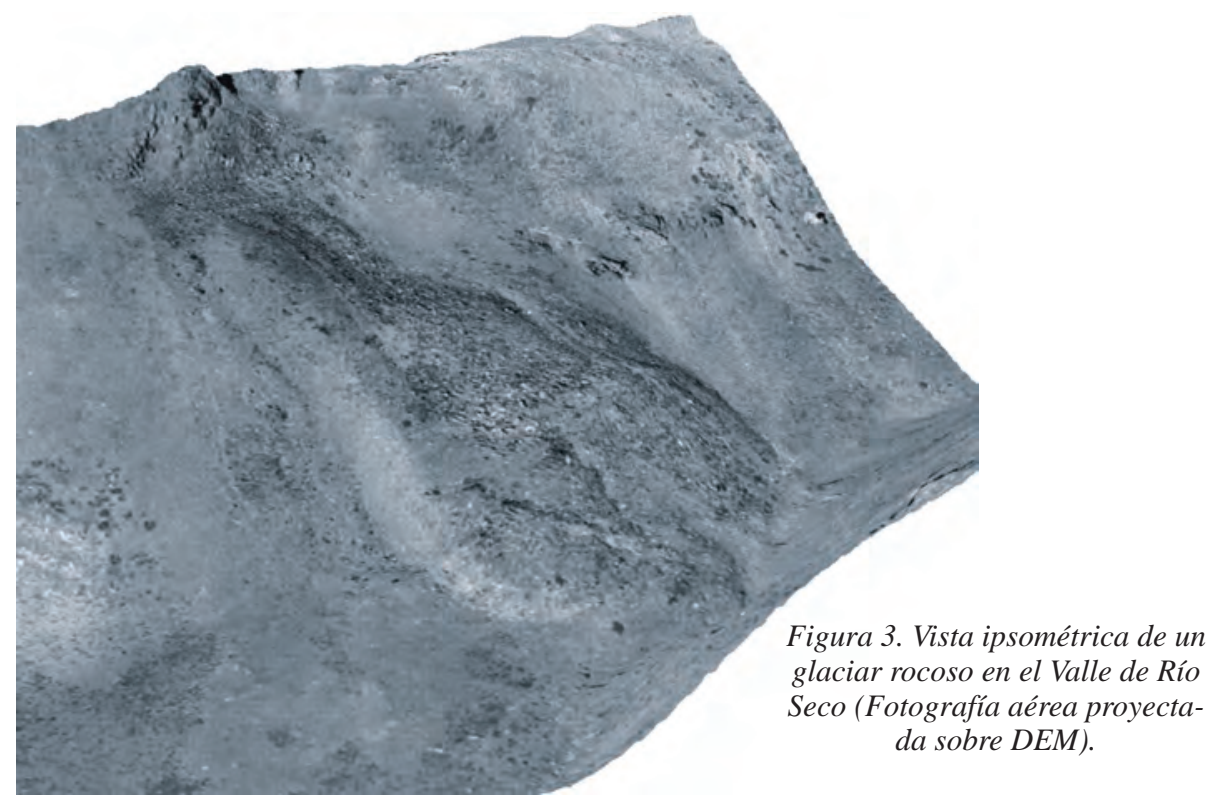

En una segunda fase, se obtuvieron datos preliminares de la cronológica de los glaciares rocosos, a partir del isótopo cosmogénico ${ }^{36} \mathrm{Cl}$. El método aplicado consistió en un primer análisis geomorfológico del área de estudio, para seleccionar los dos glaciares rocosos más representativos. En cada uno de los glaciares rocosos seleccionados, se tomaron muestras de grandes bloques estables que superaban ampliamente el metro por encima de la superficie circundante, situados en áreas cimeras de las crestas, para asegurar que nunca han sido cubiertos por sedimentos y minimizar el valor de la cubierta nival. El criterio principal para su selección era su estabilidad geomorfológica, al situarse en pendientes suaves, prácticamente sin ser afectados por la erosión. Aunque hubiera sido necesario tomar múltiples muestras en cada glaciar rocoso para obtener una datación fiable, por falta de presupuesto, únicamente se tomó una muestra en cada glaciar rocoso, o una en cada generación, en el caso que existieran varias generaciones fácilmente diferenciables por criterios geomorfológicos. Para mejorar la fiabilidad de los resultados, también se tomaron muestras de tramos distales de umbrales glaciares no alterados que reunieran las siguientes características: que conformaran resaltes, que incluyeran en su superficie restos claros de pulimento glaciar y que estuvieran situados a cotas distanciadas de los frentes de los glaciares rocosos.

Las muestras de roca, de $5 \mathrm{~cm}$ de espesor máximo, se extrajeron de las superficies horizontales de cada uno de los lugares seleccionados, con ayuda de martillo y cincel. En cuanto al protocolo seguido para la diferenciación del isótopo cosmogénico ${ }^{36} \mathrm{Cl}$ se hizo de acuerdo con los criterios propuestos por Zreda et al. (1999) y Phillips (2003). El tratamiento físico se realizó en el Laboratorio de Geografía Física de la Universidad Complutense de Madrid y en cuanto al petroquímico y análisis del Espectrómetro de Masas se ejecutó en el laboratorio de PRIME (Universidad de Purdue), y así obtener 
las proporciones de ${ }^{36} \mathrm{Cl} / \mathrm{Cl}$ y ${ }^{37} \mathrm{Cl} /{ }^{35} \mathrm{Cl}$. Por lo que respecta al análisis de la presencia de elementos U, Th, B y Gd se realizó en el laboratorio de Act Labs. El cálculo de edades se obtuvo mediante la aplicación CHLOE (Phillips and Plummer, 1996, version 3-2003), usando la escala de producción de ${ }^{36} \mathrm{Cl}$ determinada por Phillips et al. (2001) y la escala de latitud y altitud propuesta por Lal (1991). Acerca de las edades obtenidas de los glaciares rocosos analizados hay que subrayar que son provisionales y suponen sólo una primera aproximación, a la espera de ser contrastadas con nuevos análisis de otros enclaves y de aplicar otros programas más actualizados para la obtención de fechas.

\section{Resultados}

\subsection{Número y actividad de los glaciares rocosos de Sierra Nevada}

Un total de 36 glaciares rocosos han sido identificados a partir de las fotografías aéreas (Junta de Andalucía, 2005) y comprobados, posteriormente, en sucesivos trabajos de campo. Los glaciares rocosos de Sierra Nevada se desarrollaron en las zonas altas de los circos glaciares, allí donde la mayor producción de talud y la escasa radiación solar facilitaron la persistencia del permafrost discontinuo. La casi totalidad de estas zonas estuvieron beneficiadas por una superior alimentación nival (muy supeditada a los aludes y al efecto eólico sobre planicies y laderas a barlovento).

Estos glaciares rocosos, a excepción del incipiente del Corral del Veleta (Fig. 4), que aún muestra movimientos horizontales y verticales muy diferenciados (Sanjosé

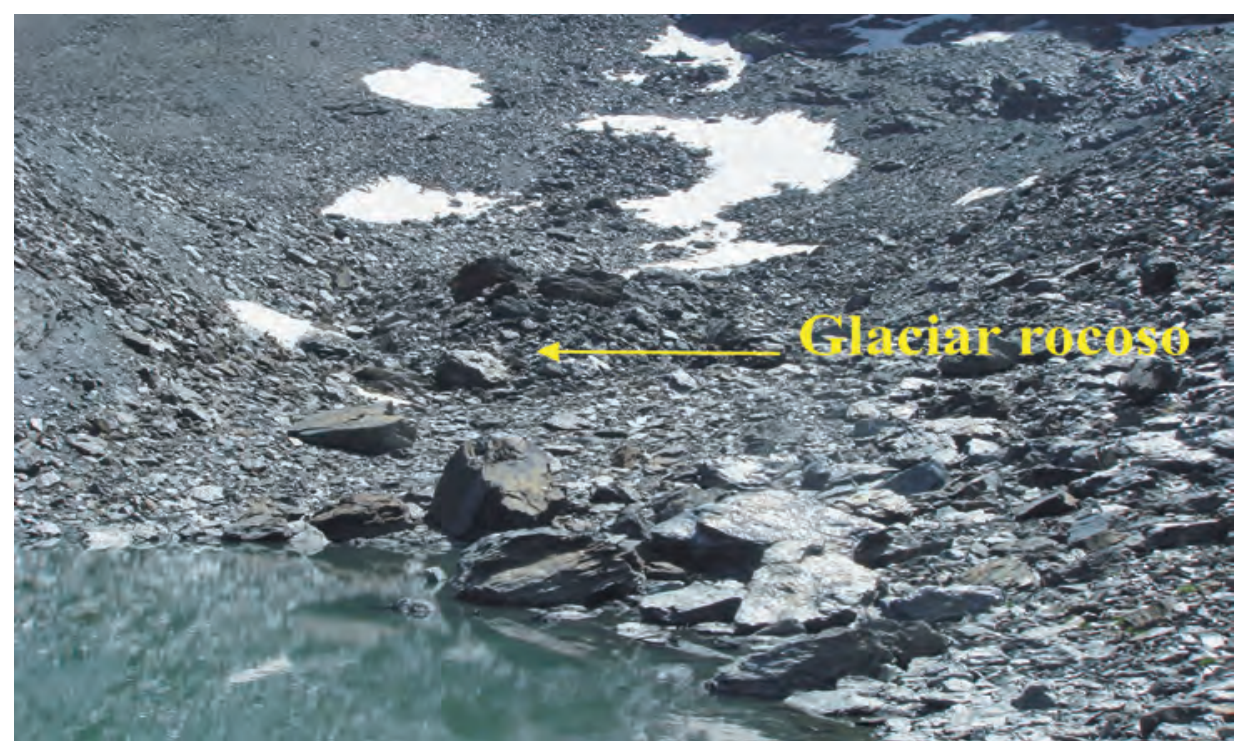

Figura 4. Frente del glaciar rocoso del Corral del Veleta. 


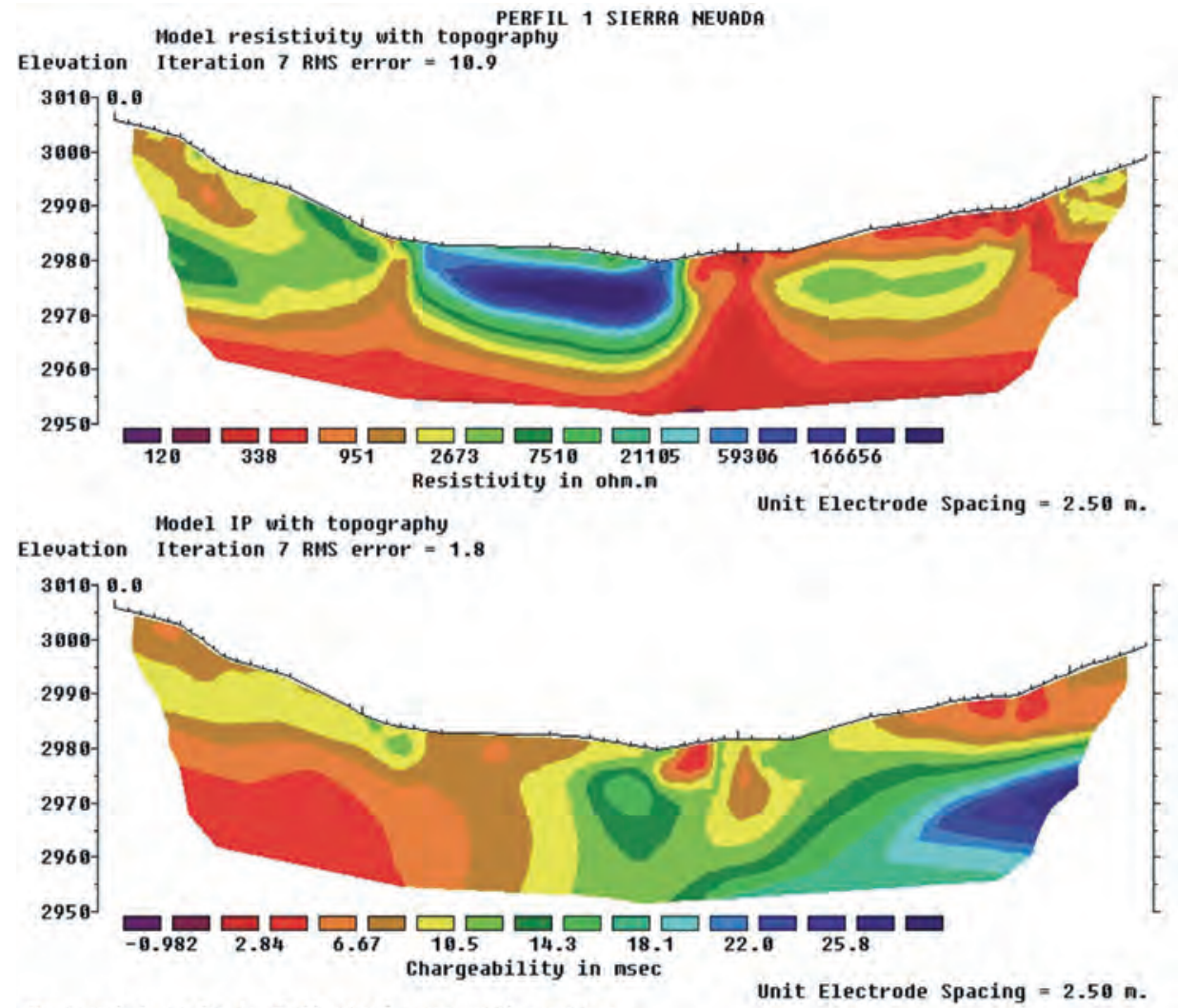

Horizontal scale is $\mathbf{8 . 9 1}$ pixels per unit spacing

Vertical exaggeration in model section display $=1.00$

First electrode is located at $0.0 \mathrm{~m}$.

Last electrode is located at $197.5 \mathrm{~m}$.

Figura 5. Tomografía del glaciar rocoso del Corral del Veleta (sección transversal N-S, agosto 2009. Realización e interpretación de J. Galindo Zaldivar).

et al., 2007), son formas relictas. No sucede así con el glaciar rocoso del Corral del Veleta, que incluye en su seno masas heladas, como se identifican en recientes estudios geofísicos realizados (Fig. 5). Sin embargo, hay que hacer notar que no se encuentra en equilibrio con las condiciones climáticas actuales, pues en su dinámica predominan los colapsos verticales por degradación de la masa helada subyacente (hielo glaciar y permafrost) frente al movimiento planar a favor de la pendiente (Gómez Ortiz et al., 2008), al contrario de lo que viene sucediendo en otros glaciares rocosos del Pirineo central y Alpes austriacos (Sanjosé et al., 2010). 


\subsection{Tamaño y localización de los glaciares rocosos de Sierra Nevada}

En su conjunto los glaciares rocosos suponen en el ámbito de la zona de estudio un $1,5 \%$ de la superficie, siempre instalados por encima de los 2400 metros de altitud. Con respecto al tamaño, el valor medio está situado en $0,08 \mathrm{~km}^{2}$, mientras que el valor máximo llega a $0,7 \mathrm{~km}^{2}$, como sucede en el caso del Cascajar del Cartujo. Acerca de la morfometría tienen, por regla general, una longitud media superior a 420 metros, oscilando entre valores mínimos de 108 metros (el caso del incipiente glaciar rocoso del Corral del Veleta) y máximos de más de $1.5 \mathrm{~km}$ como ocurre también en el Cascajar del Cartujo, que aglutina, al tiempo, una anchura máxima en torno a 700 metros (Figs. 6 y 7).

La pendiente media de la superficie de los glaciares rocosos es de $21^{\circ}$, valor muy similar a la que se indica en la mayoría de los estudios llevados a cabo en otras cadenas montañosas europeas (Barsch, 1996). En cualquier caso, existen en Sierra Nevada valores extremos que superan los $60^{\circ}$, especialmente en la parte superior de las unidades estudiadas, como por ejemplo en el caso de las formaciones lobuladas situadas en las cabeceras de los borreguiles del Dílar (inmediaciones de la Carihüela).

El cálculo de la orientación respecto a la radiación solar permite deducir, en principio, la ubicación preferente de los glaciares rocosos en enclaves que reciben poca insolación. Este hecho explica su elevado número en tal orientación, al abrigo de las empinadas cresterías que obstaculizan la radiación. Pero, al mismo tiempo, otro hecho también condiciona la proliferación de ellos, aunque ahora en orientación meridional

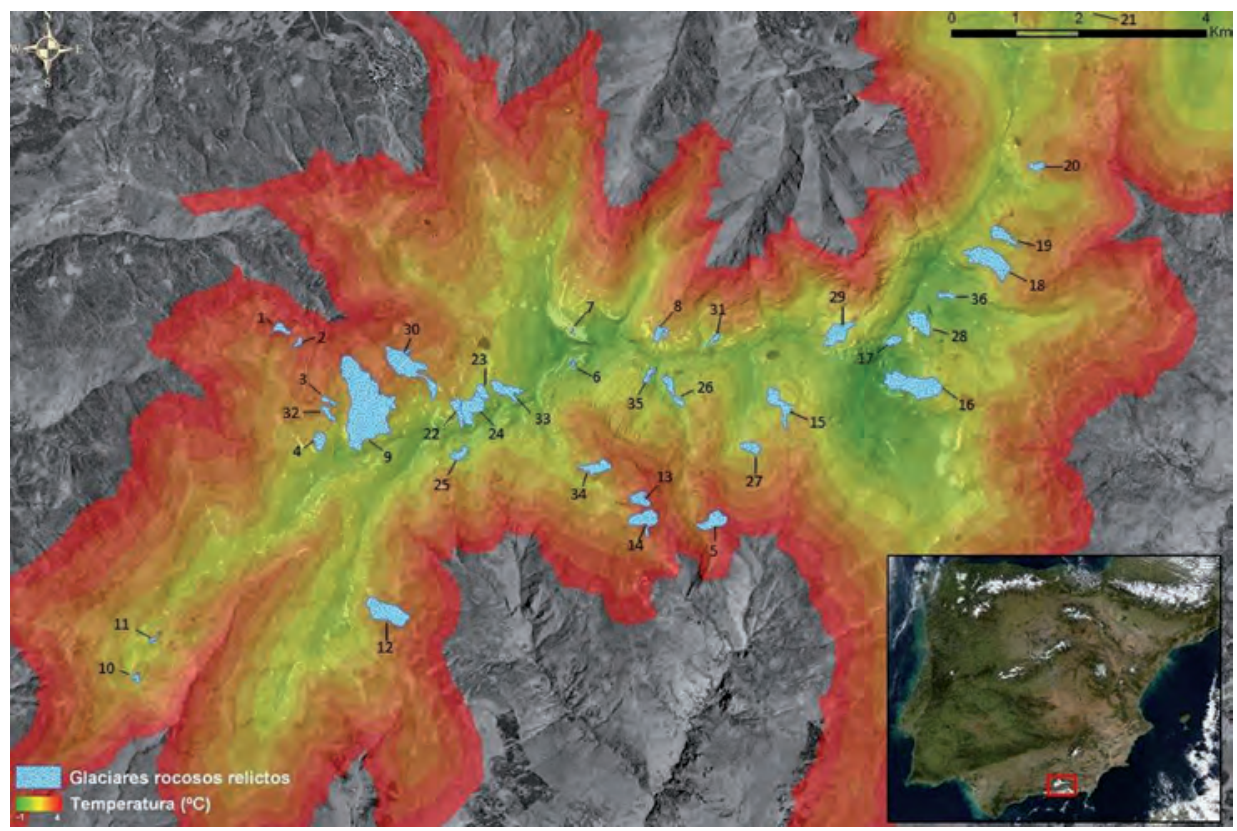

Figura 6. La localización de los glaciares rocosos en el contexto de las isotermas actuales. 


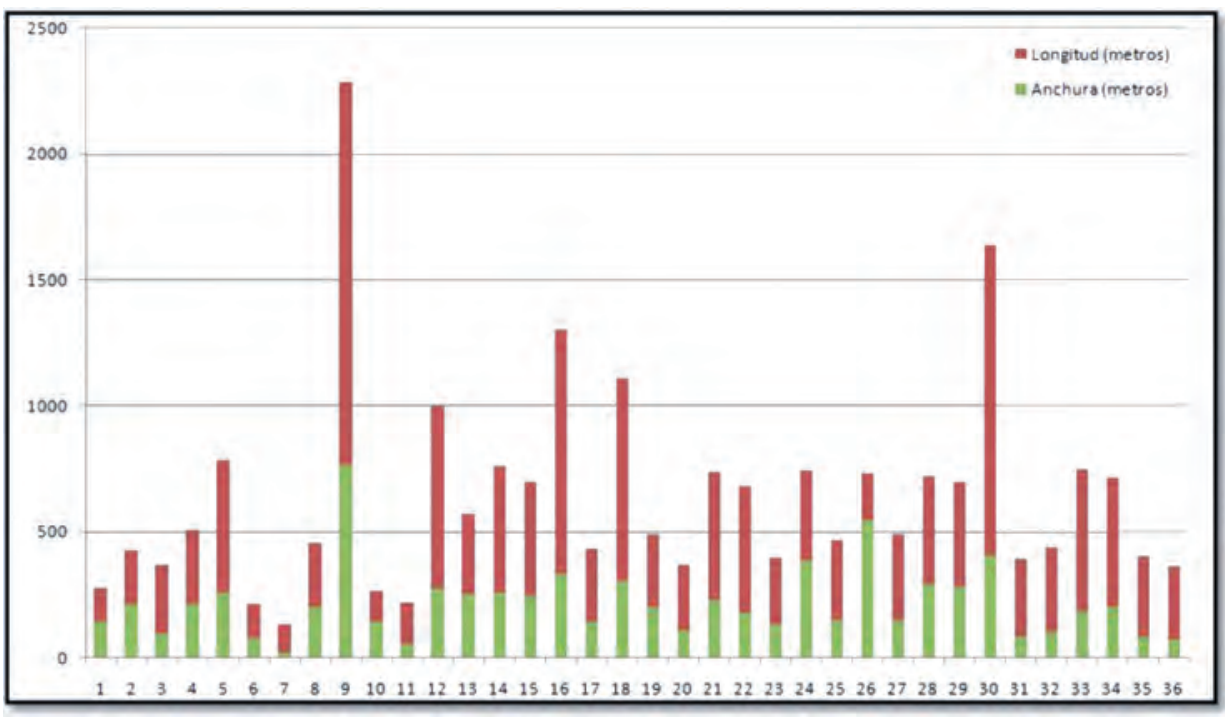

Figura 7. Longitud y anchura de los glaciares rocosos identificados.

y es la sobrealimentación nival por efecto eólico, circunstancia apuntada desde hace décadas (García Sainz, 1943; Soria y Soria, 1986; Gómez Ortiz, 1987b). En tal sentido, es conveniente señalar que más del $80 \%$ de los glaciares rocosos situados en la vertiente meridional de Sierra Nevada se encuentran en concavidades de laderas orientadas a sotavento (este o sureste) (Fig. 8). La explicación de esta circunstancia deriva de la influencia que debieron ejercer (igual que hoy) los vientos del oeste sobre las lomas y altiplanicies cimeras así como en aquellos tramos dilatados de laderas de pendiente moderada expuestos a barlovento y cuyo resultado se traduce en el barrido de nieves y

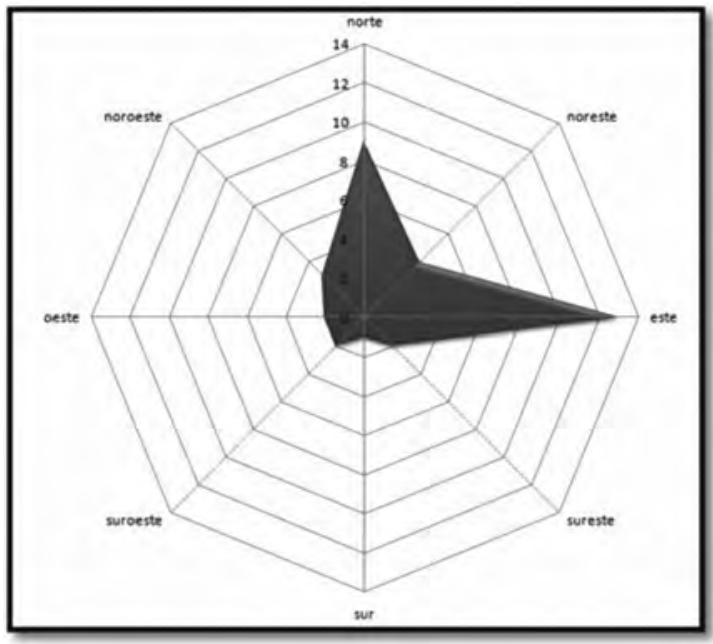

Figura 8. Orientación de los glaciares rocosos. 
en su posterior acumulación a sotavento, compensando, de esta forma, los valores altos de radiación solar característicos de la vertiente meridional (Gómez Ortiz, 1987b).

La altitud media de la raíz o inicio de los glaciares rocosos se sitúa en 2975 m de altitud, mientras que sus terminaciones o tramos distales llegan a cotas medias de $2824 \mathrm{~m}$. Los cuerpos más elevados se instalan en torno a 3360 metros (laguna Altera, en el circo de Siete Lagunas), mientras que los más bajos terminan a una altitud de $2460 \mathrm{~m}$ (valle del Dílar). Se delimita de esta manera una posible franja altitudinal de 900 metros que apunta a la posible extensión del permafrost discontinuo en la época de formación de los glaciares rocosos en Sierra Nevada y que persistiría, en términos generales, desde la retirada de los últimos glaciares pleistocenos hasta la fase de la inactividad de los glaciares rocosos. En cuanto a las condiciones térmicas de esta franja altitudinal podría caracterizarse por registrar en torno a los $-2^{\circ} \mathrm{C}$, si tenemos en cuenta que es este el valor que Barsh (1996) y Haeberli (1985), entre otros autores, señalan como temperatura media ambiental para la formación y actividad de los glaciares rocosos en los Alpes.

\subsection{Morfología de los glaciares rocosos de Sierra Nevada}

Los glaciares rocosos relictos identificados en la zona de estudio se distinguen entre sí principalmente por el tamaño, el número de crestas y surcos en superficie y por la cantidad de hielo que aglutinarían en su composición. Con respecto al último factor, la existencia de masas de hielo interno, posteriormente fundidas, queda documentado en algunas situaciones por la presencia de superficies lisas y de subsidencia, visibles, por ejemplo, en el caso del glaciar rocoso situado en la cabecera del Río del Toril (Fig. 9).

Algunas evidencias geomorfológicas y biogeográficas (morfometría, sedimentología, morfotopografía, presencia de pastizal, etc) parecen indicar la existencia de varias fases en la formación de los glaciares rocosos e, incluso, diferentes generaciones de ellos.

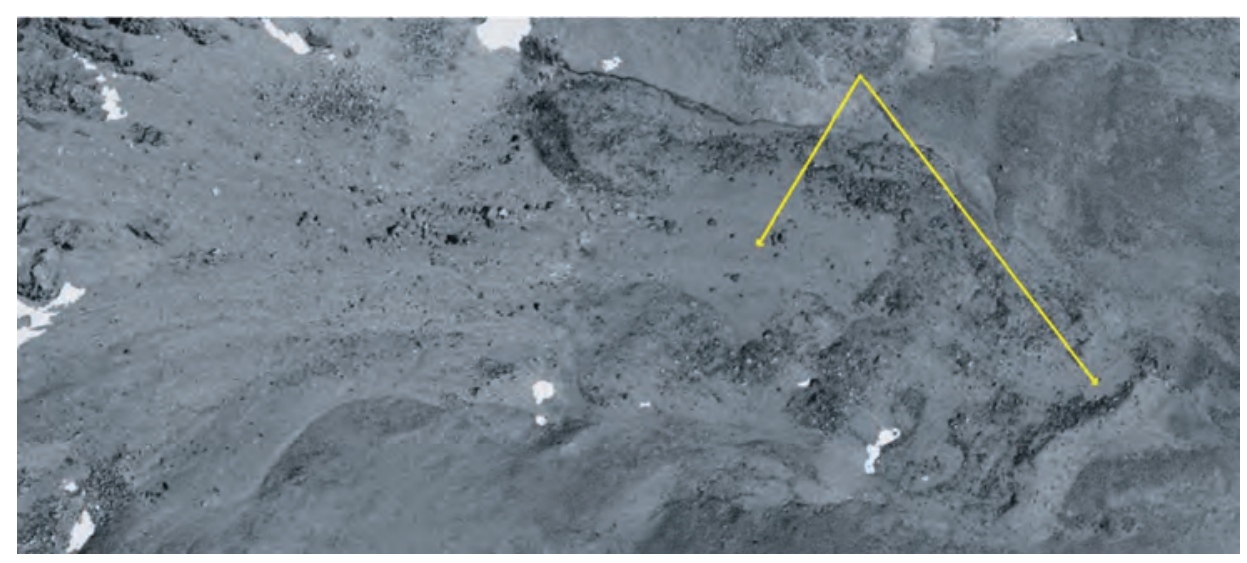

Figura 9. Áreas de subsidencia en la superficie del glaciar rocoso del Valle de Toril. 


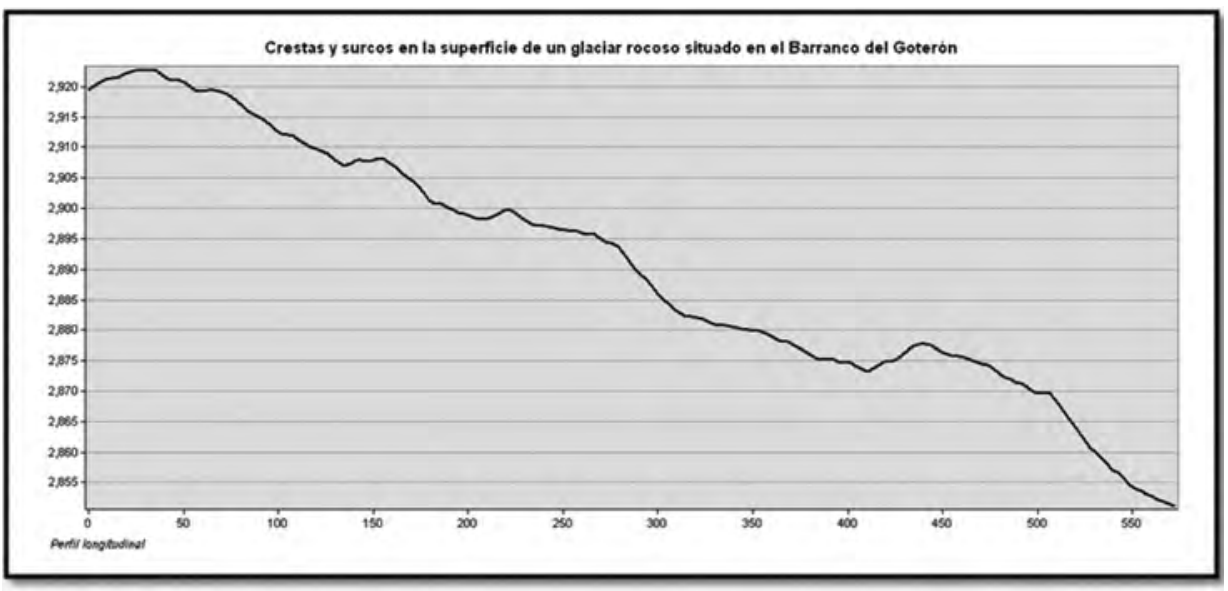

Figura 10. Crestas y surcos en la superficie de un glaciar rocoso situado en el Barranco del Goterón. Eje Y: altitud ( $m$ s.n.m); Eje X: perfil longitudial (m).

Desde el punto de vista morfológico los glaciares rocosos analizados muestran una topografía típica, caracterizada por una amalgama y sucesión de crestas y surcos (Alhorí, Siete Lagunas, Goterón, etc), arcos y lóbulos arqueados (Cascajar Negro, etc.) o amalgamas de lenguas convergentes (Corral del Veleta, etc.). Las crestas se elevan entre tres y cinco metros sobre el relieve adyacente formando ondas transversales a lo largo de la pendiente. Exceptuando formas con morfología compleja, como sucede en el Cascajar del Cartujo (valle del Dílar) con hasta seis crestas transversales, la mayoría de los glaciares rocosos de Sierra Nevada exhiben un relieve superficial en el que se distinguen dos o tres crestas transversales (Fig. 10).

\subsection{Clasificación de los glaciares rocosos de Sierra Nevada}

Tanto por los aspectos geomorfológicos como por su localización geográfica local, los glaciares rocosos de la Sierra se incluyen en la categoría de los glaciares rocosos de talud (talus rock glaciers). La mayoría se instalan en el ámbito de los circos o zonas adyacentes, siempre en el tránsito de crestas rocosas o puntales (región fuente de alimentación) y el surco colector de salida de aguas del barranco. El cuerpo del glaciar rocoso lo componen cúmulos de bloques angulosos de medidas heterométricas que incluyen en su interior abundancia de clastos menudos y arenas gruesas, además de finos. La profusión de material clástico se explica por la alta vulnerabilidad del substrato (micasquistos muy tectonizados) y por la eficacia de la gelifracción y posterior caída libre del material liberado. La explicación de la falta en el territorio de los glaciares rocosos morrénicos (debris rock glaciers) reside en las particularidades morfotopográficas y topoclimáticas de Sierra Nevada. Las pendientes acentuadas y los altos valores de energía de relieve 
impidieron la acumulación de grandes depósitos morrénicos; las temperaturas altas debieron provocar la rápida fusión tanto de los hielos de origen sedimentario, atrapados dentro de las morrenas laterales y terminales, como de las bolsas de permafrost de baja altitud.

\subsection{La edad de los glaciares rocosos de Sierra Nevada}

En primer glaciar rocoso muestreado ( $\mathrm{N}^{\mathrm{o}} 26$ de las Figs. 7 y 8 ) se localiza en la vertiente oriental de la cabecera de la garganta de Río Seco y descansa sobre un escalón rocoso, intensamente pulido por la acción de los hielos glaciares (Figs. 3, 11 y 12). Su línea de iniciación se encuentra a 3065 m s.n.m. mientras que el cuerpo del glaciar rocoso termina a unos $2940 \mathrm{~m}$. Es una forma clásica de glaciar rocoso de pared formada por la coalescencia de varios lóbulos resultando en una forma más extensa en su perfil transversal $(550 \mathrm{~m})$ que en perfil longitudinal (184 m). Cubre una superficie de 0,05 km² sobre una pendiente media de $28^{\circ}$ expuesta al este.

Por su localización, buen estado de conservación, gran estabilidad de los bloques y por el excelente estado de conservación de la superficie pulida glaciar en el umbral rocoso donde se asienta, se consideró un lugar idóneo para su datación. Entre los bloques de mayor tamaño, se seleccionó el más estable y en resalte $\left(37^{\circ} 3^{\prime} 7,252^{\prime \prime} \mathrm{N} 3^{\circ} 20^{\prime} 45,212^{\prime \prime} \mathrm{O}\right.$, $3014 \mathrm{~m}$ ), situado sobre la superficie de la tercera cresta (y última) del glaciar rocoso. El resultado de la datación fue de una antigüedad de 10.500 (+- 350) años. Además,

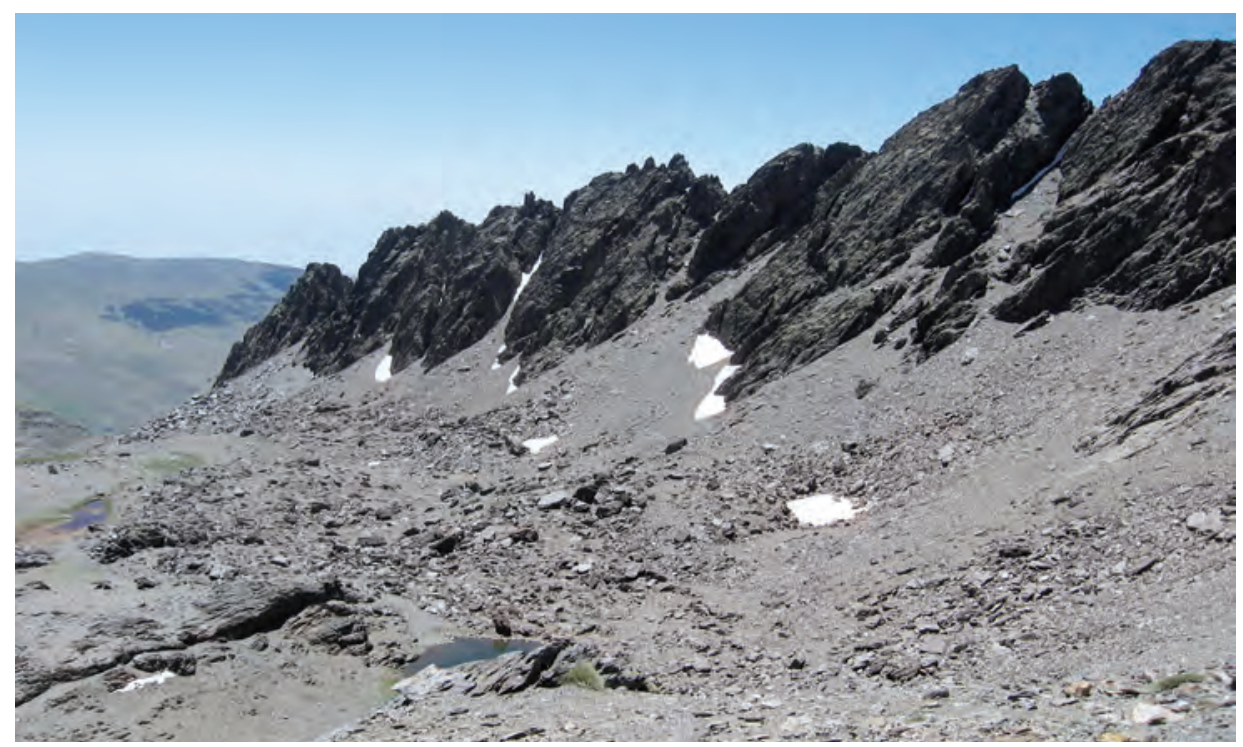

Figura 11. Glaciar rocoso de Río Seco. 


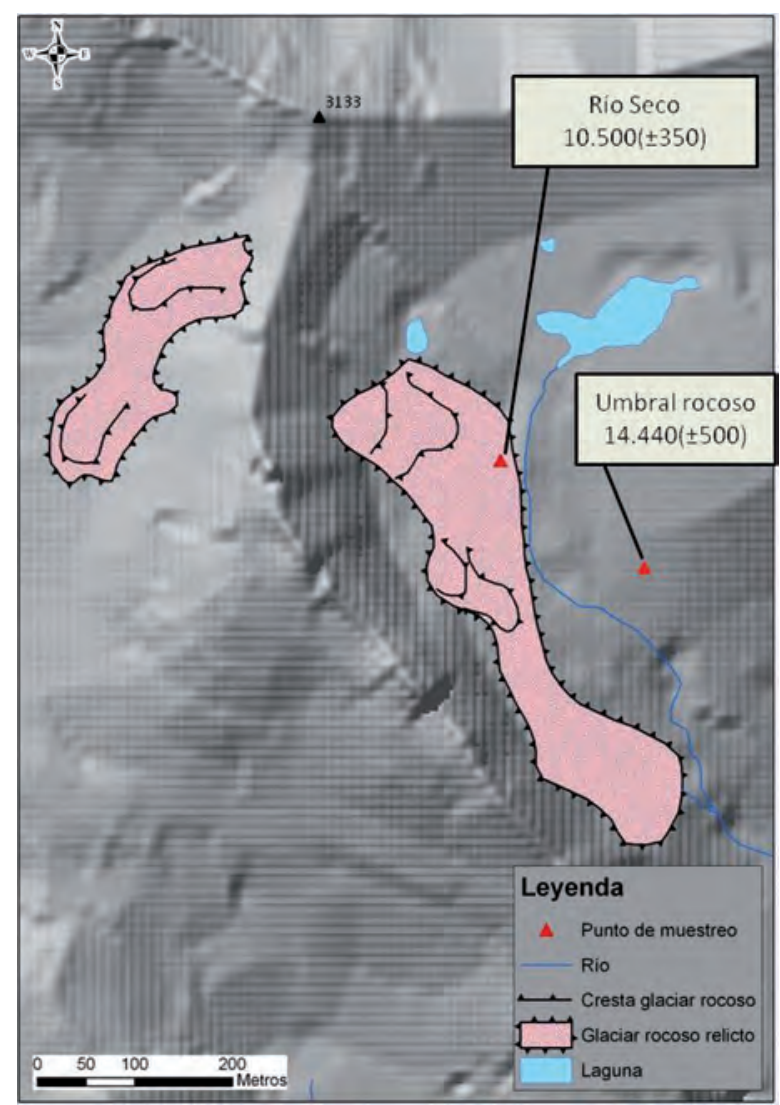

Figura 12. Esquema geomorfológico del glaciar rocoso de Río Seco y localizaciones de las muestras y resultados de las dataciones cosmogénicas $\left({ }^{36} \mathrm{Cl}\right)$.

se tomó una muestra de la superficie pulida del umbral rocoso glaciar sobre el que se asienta el glaciar rocoso (37 2' 58,03”N, 320’ 41,63”'O, $3015 \mathrm{~m}$ ). El resultado obtenido fue de una antigüedad para el umbral rocoso con pulido glaciar de 14.440 (+-500) años. Es decir, los resultados preliminares del glaciar rocosos de Río Seco indican que los hielos glaciares se retiraron entre 15 y 14 ka y que seguramente y de manera inmediata se formaron los glaciares rocosos, que quedaron inactivos hace aproximadamente $10 \mathrm{ka}$.

Para completar la información, se tomaron muestras de uno de los glaciares rocosos más grandes de la Sierra. Se trata del Cascajar del Cartujo, situado en la ladera norte del Tosal del Cartujo (3152 m), en la vertiente septentrional de la cabecera de la garganta de Dilar ( $N^{\circ} 9$ de las Figs. 7 y 8) (Figs. 13 y 14). En este caso, se han diferenciado diversas lenguas, algunas con varias generaciones de glaciares rocosos superpuestas. En la de mayor tamaño se seleccionaron muestras de dos crestas: de la más antigua, que llega hasta los $2600 \mathrm{~m}$ de altitud (muestra Cartujo 3; 37³'16,977'” 3²4'10,115'O, $2627 \mathrm{~m}$ ), y de la más reciente, cuyo frente se encuentra en los $2800 \mathrm{~m}$ (muestra Cartujo $1 ; 37^{\circ} 2^{\prime} 45,39^{\prime \prime} \mathrm{N} 3^{\circ} 24^{\prime} 9,003$ 'O, 2839 m). Por otro lado, también se tomó una muestra en 


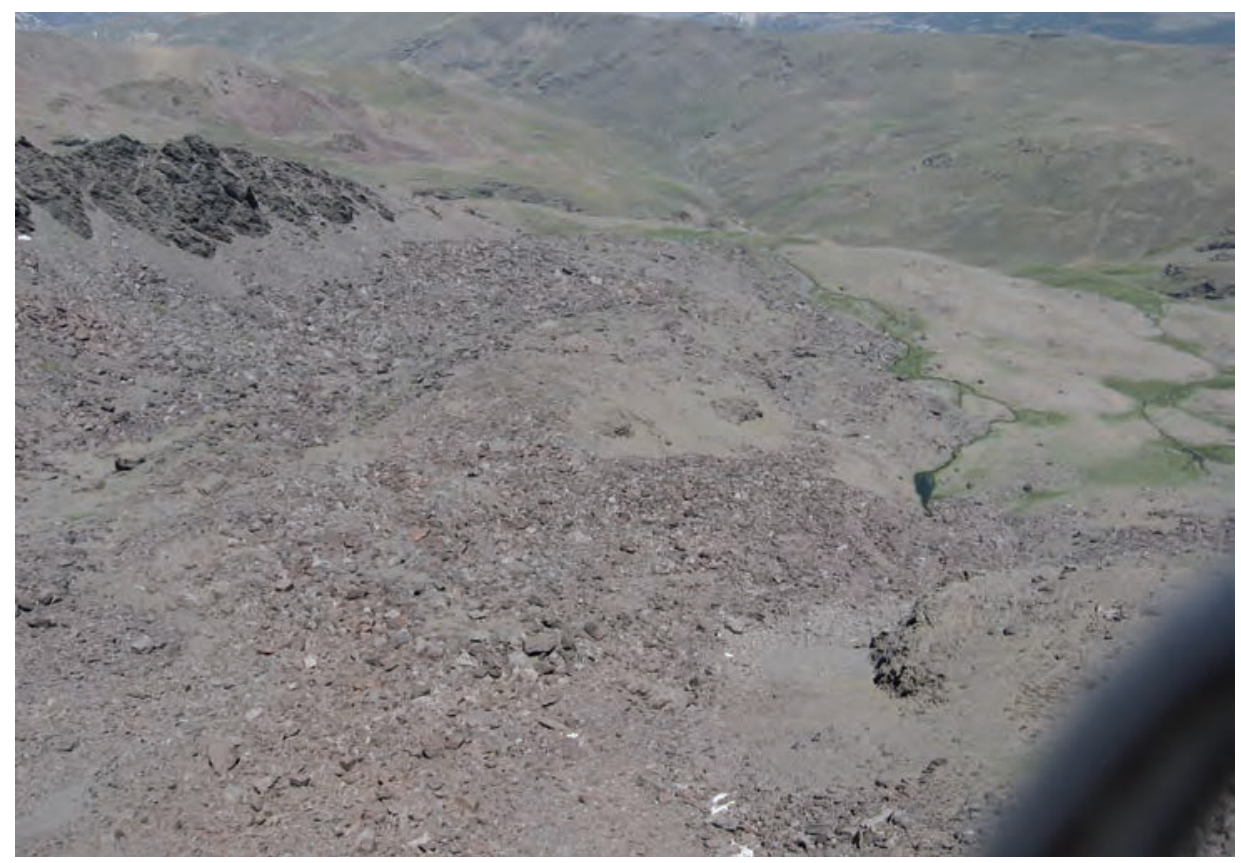

Figura 13. Glaciar rocoso del Cascajar del Cartujo.

la tercera cresta de un lóbulo algo más alejado hacía el este del cuerpo principal (muestra Cartujo 2; 37²'53,966”'N 3²3'51,26’'O, 2798 m).

Los resultados obtenidos en el Cascajar del Cartujo son muy expresivos. Cartujo1, ofrece una edad de 7.450 (+-350) años mientras que Cartujo 2, 9.650 (+- 500) años. La fecha obtenida en Cartujo 3 es de 12.900 (+-700) años. La información que aportan estas fechas es plenamente compatible con las obtenidas en Río Seco. Observamos que la fecha obtenida en la tercera cresta del glaciar rocoso de Río Seco es muy cercana a la fecha obtenida también en la tercera cresta del cuerpo de glaciar rocoso que se corresponde a la muestra Cartujo 2. Harían falta más dataciones para establecer con claridad la cronología del desarrollo de los glaciares rocosos en Sierra Nevada, pero aún así las cuatro fechas obtenidas hasta el presente parecen indicar que en condiciones climáticas estables, cada cresta necesitó un tiempo de formación cercano a mil años.

\section{Discusión}

A la luz de los resultados obtenidos, el límite inferior de la actividad de los glaciares rocosos que se conservan en Sierra Nevada se sitúa en los 2460 m de altitud. En numerosos trabajos llevados a cabo en los Alpes europeos -ver por ejemplo Barsch (1996) y 


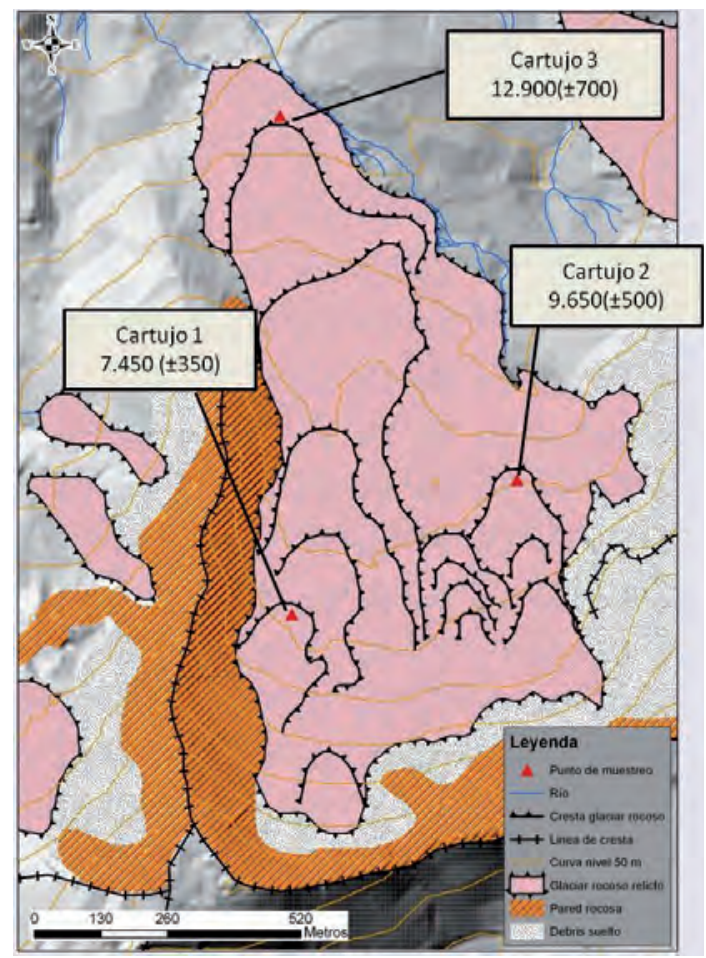

Figura 14. Esquema geomorfológico del glaciar rocoso del Cascajar del Cartujo y localizaciones de las muestras y resultados de las dataciones cosmogénicas $\left({ }^{36} \mathrm{Cl}\right)$.

Haeberli (1985)- se define una temperatura ambiental media limite de $-2^{\circ} \mathrm{C}$ para la formación y actividad de los glaciares rocosos. Siguiendo a estos trabajos, cuando extensas áreas de las cumbres de Sierra Nevada estaban cubiertas por glaciares rocosos activos, la temperatura media anual a $2460 \mathrm{~m}$ estará próxima a los $-2^{\circ} \mathrm{C}$. Sin embargo, a esa altitud en la actualidad se registra una temperatura media anual de $4^{\circ} \mathrm{C}$, es decir superior en $6^{\circ} \mathrm{C}$. Este incremento de la temperatura desde la finalización de la actividad de los glaciares rocosos hasta la actualidad es muy similar a la encontrada por Palmentola et al. (1995) en Albania $\left(6.4^{\circ} \mathrm{C}\right.$ de diferencia térmica), inferior a la encontrada en Grecia (Hughes et al., 2006) y Marruecos (Hughes et al., 2004), pero superior a los resultados encontrados en Suiza (Frauenfelder et al., 2001).

Una de las claves de esta diferencia entre los distintos estudios puede radicar en la edad de los distintos sistemas de glaciares rocosos identificados. En la actualidad existen muy pocas dataciones absolutas de glaciares rocosos. Las que se han publicado recientemente (Cosaart et al., 2010) no son comparables, ya que se tratan de glaciares rocosos mucho más recientes, aunque se producen justo después de la deglaciación de las cabeceras de los valles donde se sitúan, como en Sierra Nevada.

En este trabajo se exponen unos resultados sobre la deglaciación de Sierra Nevada derivados únicamente de una sola datación. Sin embargo, esta fecha sobre la retirada 
de los glaciares entre 14 y $15 \mathrm{ka}$ es similar a los resultados aportados recientemente en montañas ibéricas, como en los Pirineos (Pallàs et al., 2006; Delmas et al., 2008; GarcíaRuiz et al., 2010), en Gredos (Palacios et al., 2011) y también en muchos sectores de los Alpes (Ivy-Ochs et al., 2006 y 2008).

La retirada de los glaciares fue consecuencia fundamentalmente de la falta de alimentación, pero el clima seguía siendo frío, hasta $6^{\circ} \mathrm{C}$ por debajo de la media anual actual. Es evidente que inmediatamente con posterioridad a la retirada de los hielos glaciares en Sierra Nevada, se formaron los glaciares rocosos seguramente con la participación de hielo glaciar fósil, como ocurrió después de la Pequeña Edad de Hielo en el Corral del Veleta, ante la enorme actividad de las paredes deglaciarizadas de los circos.

Favorecidos por la gran producción de clastos a partir del talud y por la radiación solar reducida en los circos glaciares de altura, los glaciares rocosos nevadenses han evolucionado reptando sobre las manchas de permafrost discontinuo, incorporando, ocasionalmente, cuerpos de hielo fósil sedimentario de origen glaciar. En los casos cuando el tamaño del cuerpo de hielo incorporado ha sido de grandes dimensiones, su presencia ha quedado marcada por las superficies de subsidencia identificables, en la actualidad, en la morfología de los glaciares rocosos.

En cualquier caso, las morrenas glaciares nunca dieron lugar a la formación de glaciares rocosos, ante la escasa entidad de éstas, las fuertes pendientes y la intensidad de la erosión.

En las áreas menos favorecidas, los glaciares rocosos se paralizaron por completo y perdieron su núcleo de hielo, sólo 1 o 2 ka después de su formación. En los circos más favorables, su actividad se pudo mantener hasta 7 y 8 ka después de su formación, reflejando periodos de diversa intensidad en su actividad con la formación de varias generaciones superpuestas.

\section{Conclusiones}

Los glaciares rocosos relictos forman parte del paisaje morfológico de alta montaña que caracteriza las cumbres centro-occidentales de Sierra Nevada, entre los 2460 m y 3360 $\mathrm{m}$ de altitud. Desde el punto de vista paleoclimático son evidencias de un clima frío (presumiblemente $6^{\circ} \mathrm{C}$ de temperatura media anual menos que la actual) y de la antigua extensión del permafrost discontinuo que dominó la Sierra durante el Tardiglaciar y Holoceno inicial, desde el puerto de Trevelez hasta el cerro del Caballo, pues a oriente, en dirección al puerto de la Ragua y cerro del Almirez las condiciones ambientales imperantes de clima frío y seco no serían tan rigurosas, pues la pérdida de altitud de los cordales es notoria.

Basándonos en una primera cartografía llevada a cabo sobre fotografía aérea de alta resolución, en repetidos trabajos de campo y en los resultados de análisis morfológicos y morfométricos de las formas identificadas, los glaciares rocosos relictos analizados 
pueden clasificarse dentro de la categoría genérica de los glaciares rocosos de talud (talus rock glaciers).

Favorecidos por la gran producción de clastos a partir de la destrucción de paredes y cornisas y por los reducidos valores de radiación solar, los glaciares rocosos se formaron en las cabecera de los valles, justo después de la retirada de los hielos glaciares (hace 14 a $15 \mathrm{ka}$ ) y pudieron algunos sobrevivir hasta ya avanzado el Holoceno (entre hace 8 y 7 ka). Al final de la Pequeña Edad de Hielo se dieron unas circunstancias similares, sólo en el circo mejor dispuesto de la Sierra para la formación de glaciares rocosos, sobre todo en el Corral del Veleta. El glaciar, en su fase póstuma, quedó enterrado bajo los derrubios caídos desde la pared septentrional del Pico del Veleta y el conjunto de la masa de hielo y derrubios, gracias a unas condiciones de permafrost, adquirió capacidad de flujo.

\section{Agradecimientos}

Este trabajo se ha realizado con el soporte de los proyectos de investigación 018/2007 del Organismo Autónomo de Parques Nacionales del Ministerio de Medio Ambiente y el CSO2009-06961 del Ministerio de Ciencia e Innovación. Los autores agradecen la ayuda recibida desde el Parque Nacional de Sierra Nevada.

\section{Referencias bibliográficas}

BARSCH, D., (1996). Rock glaciers. Indicators for the present and former geoecology in high mountain environments. Springer, pp. 36, Berlin.

BOISSIER, E., (1845). Viaje botánico al sur de España durante el año 1837. Traducción de «Voyage botanique dans le midi de l’Espagne pendant l'annèe 1837». Fundación Caja Granada, 1995, Granada.

Cossart, E., Fort, M., Bourles, D., Carcaillet, J., Perrier R., Siame, L., BrauCHER, R., (2010). Climatic significance of glacier retreat and rockglaciers reassessed in the light of cosmogenic dating and weathering rind thickness in Clarée valley (Briançonnais, French Alps). Catena, 80: 204-219.

DEGENHARDT, J.J., (2009). Development of tongue-shaped and multilobate rock glaciers in alpine environments - Interpretations from ground penetrating radar surveys. Geomorphology, 109: 94-107.

Delmas, M., Gunnell, Y., Braucher, R., Calvet, M., Bourlès, D., (2008). Exposure age chronology of the last glaciation in the eastern Pyrenees. Quaternary Research, 69: 231-241. 
Díaz de Federico, A., Puga, E., Burgos, J., Gallegos, J.A., Sanz de Galdeano, C., (1980). Mapa Geológico de España. Hoja 1027, E: 1/50000 (Güejar-Sierra). IGME. Madrid.

FlÖRSChUtZ, F., MEnÉndeZ-AMOR, J., WiJMSTRA, T.A., (1971). Palynology of a thick Quaternary sucession in southern Spain. Paleography, Paleoclimatology, Paleoecology, 10: 233-264.

FRAUENFELDER, R., KÄÄB, A., (2000). Towards a paleoclimatic model of rock glacier formation in the Swiss Alps. Annals of Glaciology, 31: 281-286.

Frauenfelder, R., Haeberli, W., Hoelzle, M., Maisch, M., (2001). Using relict rock glaciers in GIS based modeling to reconstruct Younger Dryas permafrost distribution patterns in the Err-Julier area, Swiss Alps. Norskgeogr. Tidsskr., 55: 195-202.

García-Ruiz, J.M., Moreno, A., GonzÁlez Sampériz, P., Valero Garcés, B., MARTí BONO, C., (2010). La cronología del último ciclo glaciar en las montañas del sur de Europa. Una revisión. Cuaternario y Geomorfología, 24 (1-2): 35-46.

GARcía SAINZ, L., (1943). El glaciarismo cuaternario en Sierra Nevada. Estudios Geográficos, IV: 233-254.

Gómez OrTiz, A., (1987a). Contribució geomorfològica a l'estudi dels espais supraforestals pirinencs. Gènesi, organització i dinámica dels modelats glacials $i$ periglacials de la Cerdanya i l’Alt Urgell. Institut Cartogràfic de Catalunya. Barcelona.

Gómez OrTIZ, A., (1987b). Morfología glaciar en la vertiente meridional de Sierra Nevada. Estudios Geográficos, 188: 379-408.

Gómez Ortiz, A., Palacios, D., Ramos, M., Tanarro, L.M., Schulte, L., SAlvador, F., (2001). Location of permafrost in marginal regions: Corral del Veleta, Sierra Nevada, Spain. Permafrost and Periglacial Processes, 12: 93-110.

Gómez Ortiz, A., Schulte, L., SAlvador, F., SÁnchez, S., Simón, M., (2002). Mapa geomorfológico de Sierra Nevada. Morfología glaciar y periglaciar. Consejería de Medio Ambiente de la Junta de Andalucía, Parque Nacional de Sierra Nevada. Granada.

Gómez Ortiz, A., Palacios, D., Luengo, E., Tanarro, L.M., Schulte, L., Ramos, M., (2003). Talus instability in a recent deglaciation area and its relationship to buried ice and snow cover evolution (Picacho del Veleta, Sierra Nevada, Spain). Geografiska Annaler, 85 A (2): 166-182.

Gómez Ortiz, A., Plana Castellví, J. A., (2006). La Pequeña Edad del Hielo en Sierra Nevada a partir de los escritos de la época (siglos XVIII y XIX) y relaciones con el progreso de la geografía física y geomorfología española. Boletín de la Asociación de Geógrafos Españoles, 42: 71-98. 
Gómez Ortiz, A., Salvador, F., SAnjosé, J.J., Palacios, D., Schulte, L., AtKinson, A., (2008). Evolución morfodinámica de un enclave montañoso recién deglaciado: el caso del Corral del Veleta (Sierra Nevada). ¿Consecuencia del Cambio Climático? Scripta Nova-Geocrítica, Vol. XII: 270.

HAEBERLI, W., (1985). Creep of mountain permafrost: internal structure and flow of alpine rock glaciers. Mitteilungen der Versuchsanstalt für Wasserbau, Hydrologie und Glaziologie, 77: 19.

Hughes, P.D., GibBard, P.L., WoOdWARD, J.C., (2004). Quaternary glaciation in the Atlas Mountains, North Africa. En: Quaternary Glaciation - Extent and Chronology: Asia, Latin America, Africa, Australia, Antarctica (Ehlers, J., Gibbard, P.L., Eds.). Elsevier, pp. 255-260, Amsterdam.

Hughes, P.D., Gibbard, P.L., Woodward, J.C., (2006). Middle Pleistocene glacier behaviour in the Mediterranean: sedimentological evidence from the Pindus Mountains, Greece. Journal of the Geological Society, 163(5): 857-867.

Ivy-Ochs, S., Kerschner, H., Reuther, A., Maisch, M., SAIler, R., Schaefer, J., KubiK, P.W., Synal, H., SchlÜCHTER, C., (2006). The timing of glacier advances in the northern European Alps based on surface exposure dating with cosmogenic 10Be, 26Al, 36Cl, and 21Ne. En: In Situ-Produced Cosmogenic Nuclides and Quantification of Geological Processes (Siame, L.L., Bourlès, D.L., Brown, E.T., Eds.). Geological Society of America Special Paper 415, pp. 43-60.

Ivy-Ochs, S., Kerschner, H., Reuther, A., Preusser,A., Heine, K., Maisch, M., MAisch, M., KubiK, P.W., SchlÜChter, C., (2008). Chronology of the last glacial cycle in the European Alps. Journal of the Quaternary Science, 23(6-7): 559-573.

Junta de Andalucía (2005). Modelo digital del terreno de Andalucía. Relieve y orografía. Consejería de Medio Ambiente Sevilla.

KERSCHNER, H., (1983). Late glacial paleotemperatures and paleoprecipitation as derived from permafrost: glacier relationships in the Tyrolean Alps, Austria. 4th Conference on Permafrost, Fairbanks, Alaska. National Academic Press, pp. 589594, Washington.

LAL, D., (1991). Cosmic-ray labeling of erosion surfaces: in situ production rates and erosion models. Earth and Planetary Science Letters, 104: 424-439.

LHENAFF, R., (1977). Recherches géomorphologiques sur les Cordilleres Betiques centro-occidentales (Espagne). Tesis Doctoral, Université de Lille, Lille.

Messerli, B., (1965). Beiträge zur Geomorphologie der Sierra Nevada (Andalusien). Juris-Verlag, Zürich. 
Montávez, J.P., RoldÁn, C., RodríGuez, A., Jiménez, J.I., (1996). Primeros resultados de la climatología de Sierra Nevada. Conferencia Internacional Sierra Nevada. Universidad de Granada, pp. 87-99, Granada.

Palacios D., Marcos, J. VÁzQuez-Selem, L., (2011). Last Glacial Maximum and Deglaciation of Sierra de Gredos, Central Iberian Peninsula. Quaternary Internacional, 233(1): 16-26.

Pallàs, R., Rodés, A., Braucher, R., CARCAIllet, J., Ortuño, M., Bordonau, J., Bourlés, D., Vilaplana, J.M., Masana, E., SANTANACH, P., (2006). Late Pleistocene and Holocene glaciation in the Pyrenees: a critical review and new evidence from 10Be exposure ages, south-central Pyrenees. Quaternary Science Reviews, 25 : 2937-2963.

Palmentola, G., Baboci, K., GrudA, G.J., Zito, G., (1995). A note on rock glaciers in the Albanian Alps. Permafrost and Periglacial Processes, 6(3): 251-257.

PAschinger, H., (1957). Las formas glaciares de Sierra Nevada. España. Memorias y Comunicaciones del Instituto Provincial, pp. 81-94, Barcelona.

PHILLIPS, F.M., (2003). Cosmogenic 36Cl ages of Quaternary basalt flows in the Mojave Desert, California, USA. Geomorphology, 53: 199-208.

PhILliPS, F.M., Plummer, M.A., (1996). CHLOE: a program for interpreting in-situ cosmogenic nuclide data for surface exposure dating and erosion studies. $7^{\text {th }}$ International AMS Conference. Radiocarbon, 38(1): 98-99.

PhilliPs, F.M., Stone, W.D., FABRYKA-MARTin, J.T., (2001). An improved approach to calculating low-energy cosmic-ray neutron fluxes near the land/atmosphere interface. Chemical Geology, 175(3-4): 689-701.

Pons, A., ReILle, M., (1988). The Holocene and Upper Pleistocene pollen record from Padul (Granada, Spain): a new study. Paleogeography, Paleoclimatology, Paleoecology, 66: 243-267.

Puga, E., DíAz de Federico, A., Nieto, J.M., DíAz PugA, M. A., (2007). Petrología, evolución geodinámica y georrecursos del Espacio Natural de Sierra Nevada. Estudios Geológicos, 63 (2): 19-40.

Pulido Bosch, A., Pulido Bosch, M., Rodríguez Martínez, F., (1984). Consideraciones climatológicas sobre el borde occidental de Sierra Nevada (Granada). Cuad. Geogr. Univ. Granada, 12: 5-25.

Rodríguez Martínez, F., Frontana, J., Goicoichea, M., (1981). Esquema evolutivo y problemática de los estudios climatológicos sobre Sierra Nevada. VII Coloquio de Geografía, Asociación de Geógrafos Españoles, pp. 117-121, Pamplona. 
Salvador Franch, F., Gómez OrTiz, A., PAlacios, D., (2010). Comportamiento térmico del suelo en un enclave de alta montaña mediterránea con permafrost residual: Corral del Veleta (Sierra Nevada, Granada. España). En: Ambientes periglaciares, permafrost y variabilidad climática (Blanco, J.J., de Pablo, M.A., Ramos, M., Eds.), Servicio de Publicaciones Universidad de Alcalá de Henares, pp. 61-68, Alcalá de Henares.

Sanjosé, J. J., Atkinson, A., Gómez, A., Salvador Franch, F., (2007). Técnicas geodésicas y fotogramétricas aplicadas al análisis de la dinámica y cartografía del glaciar rocoso activo del Corral del Veleta (Sierra Nevada) durante el periodo 2001-2007. Mapping, 122: 26-32.

Sanjosé Blasco, J. J., Kaufmann, V., Gómez Ortiz, A., Serrano, E., AtKinson Gordo, A., Salvador Franch, F., González Trueba, J. J., (en prensa). Técnicas geomáticas aplicadas al control de los glaciares rocosos. Comparación de los glaciares rocosos de Doesen (Alpes), Posets (Pirineos) y Corral del Veleta (Sierra Nevada). Revista Cartográfica.

Serrano, E., Agudo, C., Martínez de Pisón, E., (1999). Rock glaciers in the Pyrenees. Permafrost and Periglacial Processes, 10: 101-106.

SERRAT, D., (1979). Rock glaciers morainic deposits in the eastern Pyrenees. Moraines and Varves. Balkema, pp. 93-100, Rotterdam.

Soria Mingorance, J., Soria Rodríguez, M., (1986). Depósitos de glaciares rocosos en Sierra Nevada. Acta Geológica Hispánica, 21-22: 123-129.

SOUTADÉ, G., (1980). Modelé et dinamique actuelle des versants supraforestiers des Pyrénées Orientales. Impr. Coop. Du Sud-Ouest, Albi.

Tanarro, L.M., Hoelzle, M., García, A., Ramos, M., Gruber, S., Gómez, A., PIQUeR, M., PAlacios, D., (2001). Permafrost distribution modelling in the mountains of the Mediterranean: Corral del Veleta, Sierra Nevada, Spain. Norskgeogr. Tidsskr., 55: 253-260.

Terradat-LTD \& ETH (1998). Geophysical survey report. Permafrost investigation Veleta \& Mulhacén. Sierra Nevada. PACE Unpublished Report.

Vieira, G., Ferreira, A.B., Mycielska-Dowgiallo, E., Woronko, B., OlszaK, I., (2001). Thermoluminescence Dating of Fluvioglacial Sediments (Serra da Estrela, Portugal). Actas V REQUI-I CQPLI, pp. 85-92, Lisboa.

Zreda, M., England, J., Phillips, F., Elmore, D., Sharma, P., (1999). Unblocking of the Nares Strait by Greenland and Ellesmere icesheet retreat 10,000 years ago. Nature, 398: 139-142. 